\title{
On the Number of Invariant Polynomials of Matrix Commutators
}

\author{
Enide Andrade Martins $*$ Fernando C. Silva ${ }^{\dagger \ddagger}$ \\ March 4, 1996
}

\begin{abstract}
We study the possible numbers of nonconstant invariant polynomials of the matrix commutator $X A-A X$, when $X$ varies.
\end{abstract}

Let $F$ be a field, $A, B \in F^{n \times n}$ and denote by $i(A)$ the number of nonconstant invariant polynomials of $A$.

In [7], it was proved that, if there exists $X$ such that $A+X B X^{-1}$ is nonderogatory (i. e., $i\left(A+X B X^{-1}\right)=1$ ), then $i(A)+i(B) \leq n+1$; and it was conjectured that the converse is true, under rather slight restrictions on $F$. This conjecture was proved in a theorem $[9]$ that gives necessary and sufficient conditions for the existence of $X$ such that $i\left(A+X B X^{-1}\right) \leq t$, where $t$ is a positive integer. Later [11], all the possible values of $i\left(A+X B X^{-1}\right)$, when $X$ varies, were described, assuming that $F$ is algebraically closed. In general fields, this is an open problem.

The possible numbers of nonconstant invariant polynomials of partially given matrices were also studied in several papers, e. g., $[1,4,5,10]$.

Some properties of the commutator $X A-A X$, when $X$ varies, have already been studied. Suppose that $D$ is a division ring and $A \in D^{n \times n}$. The

*Departamento de Matemática, Universidade de Aveiro, 3800 Aveiro, Portugal.

†Departamento de Matemática, Faculdade de Ciências, Universidade de Lisboa, Campo Grande, 1700 Lisboa, Portugal.

$\ddagger$ This work was partially supported by the Praxis Program (project "Álgebra e Matemáticas Discretas") and was done within the activities of the Centro de Álgebra da Universidade de Lisboa. 
rank of $X A-A X$, when $X$ runs over $D^{n \times n}$, was studied in [2]. The same problem, when $X$ runs over the set of the nonsingular matrices of $D^{n \times n}$, was studied in [8]. The eigenvalues of $X A-A X$, when $X$ runs over $F^{n \times n}$ and also when $X$ runs over the set of the nonsingular matrices of $F^{n \times n}$, were studied in $[6]$.

Given $A \in F^{n \times n}$, the following theorem solves the problem of characterizing the possible values of $i(X A-A X)$, when $X$ varies, assuming that all the irreducible polynomials in $F[x]$ have degree $\leq 2$. In particular, the problem is solved for algebraically closed fields and for the field of real numbers, $\mathbb{R}$. We shall prove Theorem 1 later. Observe that a field $F$ such that all the irreducible polynomials in $F[x]$ have degree $\leq 2$ has to be infinite.

From now on, $A$ denotes an $n \times n$ matrix over $F, f_{1}(x)|\cdots| f_{r}(x)$, $r=i(A)$, are the nonconstant invariant polynomials of $A$, and $t \in\{1, \ldots, n\}$. We assume that the invariant polynomials are always monic.

Theorem 1 Suppose that $F$ is a field such that all the irreducible polynomials in $F[x]$ have degree $\leq 2$. Let $A \in F^{n \times n}, t \in\{1, \ldots, n\}$. The following conditions are equivalent:

$\left(a_{1}\right)$ There exists a nonsingular matrix $X \in F^{n \times n}$ such that $i(X A-A X)=t$.

$\left(b_{1}\right)$ There exists $X \in F^{n \times n}$ such that $i(X A-A X)=t$.

$\left(c_{1}\right)$ One of the following conditions holds:

$\left(i_{1}\right) f_{r}(x)$ is irreducible of degree 2 and $t$ is even.

(ii $\left.i_{1}\right) f_{r}(x)$ is irreducible of degree 2 and $t \leq n / 2$.

(iii $\left.i_{1}\right) f_{r}(x)$ is not irreducible of degree 2 and $2 i(A) \leq n+t$.

Corollary 2 Suppose that $F$ is an algebraically closed field. Then $\left(a_{1}\right),\left(b_{1}\right)$ and the following condition $\left(c_{2}\right)$ are equivalent:

$\left(c_{2}\right) 2 i(A) \leq n+t$

Given a polynomial $f(x)=x^{k}-a_{k-1} x^{k-1}-\cdots-a_{1} x-a_{0}$, denote by $d(f)$ the degree of $f$ and denote by $C(f)$ the companion matrix

$$
\left[\begin{array}{c|ccc}
0 & \multicolumn{2}{|c}{I_{k-1}} \\
\hline a_{0} & a_{1} & \cdots & a_{k-1}
\end{array}\right] .
$$


Let $\bar{F}$ be an algebraically closed extension of $F$. Let

$$
R_{\bar{F}}(A)=\min _{\lambda \in \bar{F}} \operatorname{rank}\left(A-\lambda I_{n}\right)
$$

Lemma $3[7] R_{\bar{F}}(A)=n-i(A)$.

It is well-known that $A$ is nonderogatory if and only if $i(A)=1$. It follows, from the previous lemma, that $A$ is nonderogatory if and only if $R_{\bar{F}}(A)=n-1$.

Lemma $4[2,8]$ Suppose that either $F \neq\{0,1\}$ or $n \neq 2$. Let $\rho \in\{0, \ldots, n\}$. The following statements are equivalent:

$\left(a_{4}\right)$ There exists a nonsingular matrix $X \in F^{n \times n}$ such that $\operatorname{rank}(X A-$ $A X)=\rho$.

$\left(b_{4}\right)$ There exists $X \in F^{n \times n}$ such that $\operatorname{rank}(X A-A X)=\rho$.

$\left(c_{4}\right)$ One of the following conditions holds:

$\left(i_{4}\right) f_{r}(x)$ is irreducible of degree 2 and $\rho$ is even.

(ii $\left.i_{4}\right) f_{r}(x)$ is irreducible of degree $\geq 3$ and $\rho \neq 1$.

$\left(\right.$ iii $\left._{4}\right) f_{r}(x)$ is not irreducible of degree $\geq 2$ and $\rho \leq 2 R_{\bar{F}}(A)$.

Remark 1 In the original papers, Lemma 4 was established, with a slightly different statement, for arbitrary division rings. More precisely, [2] gives a necessary and sufficient condition for $\left(b_{4}\right)$ and [8] gives a necessary and sufficient condition for $\left(a_{4}\right)$.

Lemma 5 [6] Let $c_{1}, \ldots, c_{n}$ be elements of $F$ such that $c_{1}+\cdots+c_{n}=0$. If $2 i(A) \leq n$ and $d\left(f_{r}\right) \geq 3$, then there exists a nonsingular matrix $X \in F^{n \times n}$ such that $X A-A X$ has eigenvalues $c_{1}, \ldots, c_{n}$.

If $A^{\prime} \in F^{n \times n}$ is similar to $A$ and $Z \in F^{n \times n}$ is a nonsingular matrix such that $A^{\prime}=Z^{-1} A Z$, then $R_{\bar{F}}(A)=R_{\bar{F}}\left(A^{\prime}\right)$ and, for every $X \in F^{n \times n}, X A-A X$ and $\left(Z^{-1} X Z\right) A^{\prime}-A^{\prime}\left(Z^{-1} X Z\right)$ are similar. Therefore, in the proofs of the 
following lemmas and in the proof of Theorem 1, we can replace $A$ by any similar matrix and we shall assume, without loss of generality, that

$$
A=C\left(f_{1}\right) \oplus \cdots \oplus C\left(f_{r}\right)
$$

Assume that $A$ has the form (1). Then, for every $\lambda \in \bar{F}, \operatorname{rank}\left(A-\lambda I_{n}\right) \geq$ $n-r$. The equality holds if and only if $\lambda$ is a root of $f_{1}(x)$. Note that this argument gives a proof for Lemma 3. The eigenvalues of $A$ are the roots of $f_{1}(x) \cdots f_{r}(x)$. We call primary eigenvalues of $A$ to the roots of $f_{1}(x)$.

If $A$ has a primary eigenvalue $\lambda \in F$, then $R_{\bar{F}}(A)=R_{\bar{F}}\left(A-\lambda I_{n}\right)$ and, for every $X \in F^{n \times n}, X A-A X=X\left(A-\lambda I_{n}\right)-\left(A-\lambda I_{n}\right) X$. Therefore, in the proofs of the following lemmas and in the proof of Theorem 1, if $A$ has a primary eigenvalue in $F$, we shall also assume, without loss of generality, that 0 is a primary eigenvalue of $A$. Note that, in this case, the first column of $C\left(f_{i}\right), i \in\{1, \ldots, r\}$, is equal to zero.

Corollary 6 Suppose that $F$ is an infinite field. Let $c \in F$. If $n \geq 4,2 i(A) \leq$ $n$ and $d\left(f_{r}\right) \geq 3$, then there exists a nonsingular matrix $X \in F^{n \times n}$ such that $X A-A X$ is nonderogatory and $c$ is eigenvalue of $X A-A X$.

Proof. As $F$ is infinite and $n \geq 4$, there exist distinct elements $c_{1}, \ldots, c_{n} \in$ $F$ such that $c_{1}=c$ and $c_{1}+\cdots+c_{n}=0$. According to Lemma 5 , there exists a nonsingular matrix $X \in F^{n \times n}$ such that $X A-A X$ has eigenvalues $c_{1}, \ldots, c_{n}$. Clearly, $X A-A X$ is nonderogatory.

The following corollary is also easy to prove.

Corollary 7 Suppose that $F$ is an infinite field. Let $G$ be a finite subset of $F$. If $2 i(A) \leq n$ and $d\left(f_{r}\right) \geq 3$, then there exists a nonsingular matrix $X \in F^{n \times n}$ such that $X A-A X$ is nonderogatory and does not have any eigenvalue in $G$.

Lemma 8 Suppose that $F$ is an infinite field. If $C \in F^{2 \times 2}$ is nonderogatory, then the set of the values $\operatorname{det}(X C-C X)$, where $X$ runs over the set of the nonsingular matrices of $F^{2 \times 2}$ such that $X C-C X$ is nonderogatory, is infinite. 
Proof. Without loss of generality, suppose that

$$
C=\left[\begin{array}{ll}
0 & 1 \\
a & b
\end{array}\right] .
$$

Choose $u \in F \backslash\{0,1\}$. For every $v \in F$, let

$$
X_{v}=\left[\begin{array}{cc}
u-1 & 0 \\
v & u
\end{array}\right] .
$$

Then $X_{v}$ is nonsingular, $X_{v} C-C X_{v}$ is nonderogatory and $\operatorname{det}\left(X_{v} C-C X_{v}\right)=$ $-v^{2}-b v+a$. For every $\lambda \in F$, the quadratic equation, in $v,-v^{2}-b v+a=\lambda$ has, at most, 2 roots. Therefore $\left\{-v^{2}-b v+a: v \in F\right\}$ is infinite.

Lemma 9 Suppose that $F$ is an infinite field. Let $G$ be a finite subset of $\bar{F}$. If $d\left(f_{1}\right)=\cdots=d\left(f_{r}\right)=2$, then there exists a nonsingular matrix $X \in F^{n \times n}$ such that $X A-A X$ is nonderogatory and does not have any eigenvalue in G.

Proof. We have $f_{1}=\cdots=f_{r}$. Let $C=C\left(f_{1}\right)$. It follows, from Lemma 8 , that there exists an infinite list of nonsingular matrices $X_{1}, X_{2}, \ldots$, in $F^{2 \times 2}$, such that $D_{i}:=X_{i} C-C X_{i}$ is nonderogatory and $\operatorname{det} D_{i} \neq \operatorname{det} D_{j}$, $i, j \in\{1,2, \ldots\}, i \neq j$. As all the matrices $D_{i}$ have trace equal to zero, it is easy to deduce that, if $i \neq j$, then $D_{i}$ and $D_{j}$ do not have a common eigenvalue. Without loss of generality, we may assume that none of the matrices $D_{1}, \ldots, D_{r}$ has an eigenvalue in $G$. Then $X A-A X$, where $X=$ $X_{1} \oplus \cdots \oplus X_{r}$, is nonderogatory and does not have any eigenvalue in $G$.

Lemma 10 Suppose that $F \neq\{0,1\}$. Let $\rho \in\{2, \ldots, n-1\}$. If $A$ is nonderogatory, then there exists a nonsingular matrix $X \in F^{n \times n}$ such that $X A-A X$ is nilpotent and $R_{\bar{F}}(X A-A X)=\rho$.

Proof. According to a previous assumption, $A=C\left(f_{1}\right)$. If $\rho$ is even, let $X$ be the $n \times n$ matrix with the principal entries, and the entries $(2 k+1,2 k-1)$, $k \in\{1, \ldots, \rho / 2\}$, equal to 1 .

If $\rho$ is odd, let $e \in F \backslash\{0,1\}$ and let $X$ be the $n \times n$ matrix with the principal entries, and the entries $(2 k+1,2 k-1), k \in\{1, \ldots,(\rho-1) / 2\}$, equal to 1 and the entry $(\rho+1, \rho-1)$ equal to $e$. 
For any value of $\rho, X A-A X$ is lower triangular with the principal entries equal to zero, the entries $(i+1, i), i \in\{1, \ldots, \rho\}$, different from zero, and the columns $\rho+1, \ldots, n$ equal to zero. Clearly, $X A-A X$ is nilpotent and $R_{\bar{F}}(X A-A X)=\rho$.

Lemma 11 Suppose that $F$ is an infinite field. Let $\rho \in\{0, \ldots, n-1\}$. Condition $\left(c_{4}\right)$ is equivalent to any of the following conditions:

$\left(a_{11}\right)$ There exists a nonsingular matrix $X \in F^{n \times n}$ such that $R_{\bar{F}}(X A-$ $A X)=\operatorname{rank}(X A-A X)=\rho$.

$\left(b_{11}\right)$ There exists $X \in F^{n \times n}$ such that $R_{\bar{F}}(X A-A X)=\operatorname{rank}(X A-A X)=$ $\rho$.

Proof. We only need to prove that $\left(c_{4}\right)$ implies $\left(a_{11}\right)$. This proof is by induction on $n$. Suppose that $\left(c_{4}\right)$ is satisfied. Note that, if $\rho=0$, then $\left(a_{11}\right)$ is trivial. Thus, suppose that $\rho>0$. Then $\left(c_{4}\right)$ implies that $A$ is nonscalar.

Suppose that $\rho=1$. According to Lemma 4, there exists a nonsingular matrix $X \in F^{n \times n}$ such that $\operatorname{rank}(X A-A X)=1$. Then $R_{\bar{F}}(X A-A X)=1$.

We have already proved Lemma 11 when $n \leq 2$.

Suppose that $n \geq 3$ and that $\rho \geq 2$. The case $r=1$ follows, immediately, from Lemma 10. Then, we also suppose that $r \geq 2$.

Case 1. Suppose that $\rho=n-1$.

Subcase 1.1. Suppose that $d\left(f_{r}\right) \geq 3$. As $r \geq 2$, we have $n \geq 4$. From $\left(c_{4}\right)$, it follows that $2 i(A) \leq n+1$. If $2 i(A) \leq n$, then, according to Corollary 6 , there exists a nonsingular matrix $X \in F^{n \times n}$ such that $X A-A X$ is nonderogatory and 0 is eigenvalue of $X A-A X$, that is, $\left(a_{11}\right)$ is satisfied, with $\rho=n-1$.

Now suppose that $2 i(A)=n+1$. Then $f_{1}(x)$ has degree 1 and $A=[c] \oplus A_{0}$, where $c \in F$ and $A_{0}=C\left(f_{2}\right) \oplus \cdots \oplus C\left(f_{r}\right)$. According to Corollary 7, there exists a nonsingular matrix $X_{0} \in F^{(n-1) \times(n-1)}$ such that $X_{0} A_{0}-A_{0} X_{0}$ is nonderogatory and nonsingular. Taking $X=[1] \oplus X_{0}, X A-A X$ is nonderogatory and 0 is eigenvalue of $X A-A X$, that is, $\left(a_{11}\right)$ is satisfied.

Subcase 1.2. Suppose that $d\left(f_{r}\right)=2$. As $\left(c_{4}\right)$ is satisfied, $f_{r}(x)$ is reducible and, therefore, $A$ has a primary eigenvalue in $F$. According to a previous assumption, 0 is a primary eigenvalue of $A$.

Note that, if $n$ is even, then $r=n / 2$ and $f_{1}(x)=\cdots=f_{r}(x)$; and, if $n$ is odd, then $r=(n+1) / 2, f_{1}(x)=x$ and $f_{2}(x)=\cdots=f_{r}(x)$. 
Let $A_{1}=C\left(f_{1}\right)$ and $A_{2}=C\left(f_{2}\right) \oplus \cdots \oplus C\left(f_{r}\right)$. If $n$ is even, then there exists, as we have already seen, a nonsingular matrix $X_{1} \in F^{2 \times 2}$ such that $R_{\bar{F}}\left(X_{1} A_{1}-A_{1} X_{1}\right)=\operatorname{rank}\left(X_{1} A_{1}-A_{1} X_{1}\right)=1$. If $n$ is odd, let $X_{1}=[1] \in F^{1 \times 1}$.

In any case, according to Lemma 9 , there exists a nonsingular matrix $X_{2} \in F^{n^{\prime} \times n^{\prime}}$, where $n^{\prime}$ is the largest even integer less than $n$, such that $X_{2} A_{2}-A_{2} X_{2}$ is nonderogatory and does not have any eigenvalue in common with $X_{1} A_{1}-A_{1} X_{1}$.

Let $X=X_{1} \oplus X_{2}$. Then $R_{\bar{F}}(X A-A X)=\operatorname{rank}(X A-A X)=n-1$.

Case 2. Suppose that $\rho<n-1$. Let $\delta=d\left(f_{1}\right), \rho_{1}=\min \{\delta-1, \rho\}$, $\rho_{2}=\rho-\rho_{1}, A_{1}=C\left(f_{1}\right), A_{2}=C\left(f_{2}\right) \oplus \cdots \oplus C\left(f_{r}\right)$. Then $R_{\bar{F}}\left(A_{1}\right)=\delta-1$ and $R_{\bar{F}}\left(A_{2}\right)=R_{\bar{F}}(A)-\delta+1$. If $\delta=1$, we have $2 R_{\bar{F}}\left(A_{2}\right)=2 R_{\bar{F}}(A) \geq \rho=\rho_{2}$. If $\delta>1$ and $\rho_{1}=\delta-1$, we have $2 R_{\bar{F}}\left(A_{2}\right) \geq n-\delta>\rho-\delta+1=\rho_{2}$. If $\rho_{1}=\rho$, then $\rho_{2}=0$ and we also have $2 R_{\bar{F}}\left(A_{2}\right) \geq \rho_{2}$.

If the induction assumption can be used, then there exist nonsingular matrices $X_{1} \in F^{\delta \times \delta}, X_{2} \in F^{(n-\delta) \times(n-\delta)}$ such that $R_{\bar{F}}\left(X_{1} A_{1}-A_{1} X_{1}\right)=$ $\operatorname{rank}\left(X_{1} A_{1}-A_{1} X_{1}\right)=\rho_{1}$ and $R_{\bar{F}}\left(X_{2} A_{2}-A_{2} X_{2}\right)=\operatorname{rank}\left(X_{2} A_{2}-A_{2} X_{2}\right)=\rho_{2}$. Let $X=X_{1} \oplus X_{2}$. Clearly, $R_{\bar{F}}(X A-A X)=\operatorname{rank}(X A-A X)=\rho$.

Now suppose that the induction assumption cannot be used in the previous argument. Then one, at least, of the following conditions is satisfied:

$\left(a_{11}^{\prime}\right) f_{1}(x)$ is irreducible and $\rho_{1}=1$.

$\left(b_{11}^{\prime}\right) f_{r}(x)$ is irreducible and $\rho_{2}=1$.

Subcase 2.1. Suppose that $\left(a_{11}^{\prime}\right)$ is satisfied.

Subcase 2.1.1. Suppose that $\rho<n-2$. Note that, if $\rho_{1}=\rho$, then $\left(c_{4}\right)$ implies that $f_{r}(x)$ is reducible; and that, if $\rho_{1}=\delta-1<\rho$ and $\rho$ is odd, then $\left(c_{4}\right)$ also implies that $f_{r}(x)$ is reducible. In any situation, according to the induction assumption, there exists a nonsingular matrix $X_{2} \in F^{(n-\delta) \times(n-\delta)}$ such that $R_{\bar{F}}\left(X_{2} A_{2}-A_{2} X_{2}\right)=\operatorname{rank}\left(X_{2} A_{2}-A_{2} X_{2}\right)=\rho$. Let $X=I_{\delta} \oplus X_{2}$. Then $R_{\bar{F}}(X A-A X)=\operatorname{rank}(X A-A X)=\rho$.

Subcase 2.1.2. Suppose that $\rho=n-2$. Then $1=\rho_{1}=\delta-1<\rho$. According to Corollary 7 or Lemma 9 , there exists a nonsingular matrix $X_{2} \in F^{(n-2) \times(n-2)}$ such that $X_{2} A_{2}-A_{2} X_{2}$ is nonderogatory and 0 is not eigenvalue of $X_{2} A_{2}-A_{2} X_{2}$. Let $X=I_{\delta} \oplus X_{2}$. Then $R_{\bar{F}}(X A-A X)=$ $\operatorname{rank}(X A-A X)=\rho$.

Subcase 2.2. Suppose that $\left(a_{11}^{\prime}\right)$ is false and that $\left(b_{11}^{\prime}\right)$ is satisfied. Then $1<\rho_{1}=\delta-1<\rho$. 
Firstly, suppose that $\rho_{1}>2$. According to the induction assumption, there exist nonsingular matrices $X_{1} \in F^{\delta \times \delta}$ and $X_{2} \in F^{(n-\delta) \times(n-\delta)}$ such that $R_{\bar{F}}\left(X_{1} A_{1}-A_{1} X_{1}\right)=\operatorname{rank}\left(X_{1} A_{1}-A_{1} X_{1}\right)=\rho-2$ and $R_{\bar{F}}\left(X_{2} A_{2}-\right.$ $\left.A_{2} X_{2}\right)=\operatorname{rank}\left(X_{2} A_{2}-A_{2} X_{2}\right)=2$. Let $X=X_{1} \oplus X_{2}$. Then $R_{\bar{F}}(X A-A X)=$ $\operatorname{rank}(X A-A X)=\rho$.

Now suppose that $\rho_{1}=2$. According to Corollary 7 , there exists a nonsingular matrix $X_{1} \in F^{3 \times 3}$ such that $X_{1} A_{1}-A_{1} X_{1}$ is nonderogatory and 0 is not eigenvalue of $X_{1} A_{1}-A_{1} X_{1}$. Let $X=X_{1} \oplus I_{n-3}$. Then $R_{\bar{F}}(X A-A X)=$ $\operatorname{rank}(X A-A X)=3=\rho$.

Now suppose that $C \in F^{2 \times 2}$ is a matrix of the form (2) and that the characteristic polynomial of $C$ is irreducible. According to [3], $K=F[C]$ is a field and, according to [2], there exists $B \in F^{2 \times 2}$ such that $U=B C-C B$ is nonsingular and

$$
\left\{X C-C X \mid X \in F^{2 \times 2}\right\}=U K=K U .
$$

Let $S=\left[S_{i, j}\right] \in \bar{F}^{2 p \times 2 p}$, where the blocks $S_{i, j}$ are of size $2 \times 2$. We shall say that $S$ is a $\star$-matrix if $S_{i, j} \in U K$, whenever $i \leq j$.

Lemma 12 With the previous notation, suppose that $b \neq 0$. Let $S=\left[S_{i, j}\right] \in$ $\bar{F}^{2 p \times 2 p}, S_{i, j} \in \bar{F}^{2 \times 2}$, be $a \star$-matrix and $\lambda \in \bar{F} \backslash\{0\}$. Then $\operatorname{rank}\left(S-\lambda I_{2 p}\right) \geq p$.

Proof. By induction on $p$. Firstly, note that a commutator $X C-C X$ is not scalar, unless it is the zero matrix. Therefore, the lemma is true when $p=1$.

Suppose that $p \geq 2$. Suppose that $S_{1, j}=0$, for every $j \in\{2, \ldots, p\}$. Considering $S$ as a $p \times p$ matrix with entries in $\bar{F}^{2 \times 2}$, let $S_{0}$ be the principal submatrix obtained from $S$ by deleting the first row and the first column. Using the induction assumption,

$$
\operatorname{rank}\left(S-\lambda I_{2 p}\right) \geq \operatorname{rank}\left(S_{1,1}-\lambda I_{2}\right)+\operatorname{rank}\left(S_{0}-\lambda I_{2 p-2}\right) \geq p .
$$

Now suppose that $S_{1, j} \neq 0$, for some $j \in\{2, \ldots, p\}$. Choose the maximum $v \in\{2, \ldots, p\}$ such that $S_{1, v} \neq 0$. Consider $S-\lambda I_{2 p}$ as a $p \times p$ matrix with entries in $\bar{F}^{2 \times 2}$. For each $w \in\{2, \ldots, v-1\}$, subtract the $v$ th column multiplied by $S_{1, v}^{-1} S_{1, w}$ from the $w$ th column. It is not hard to see that the matrix obtained has the form $S^{\prime}-\lambda I_{2 p}$, where $S^{\prime}=\left[S_{i, j}^{\prime}\right], S_{i, j}^{\prime} \in \bar{F}^{2 \times 2}$, is a $\star$-matrix. Clearly, $\operatorname{rank}\left(S-\lambda I_{2 p}\right)=\operatorname{rank}\left(S^{\prime}-\lambda I_{2 p}\right)$. If $p=2$, then 
$\operatorname{rank}\left(S^{\prime}-\lambda I_{4}\right) \geq \operatorname{rank} S_{1,2}^{\prime}=2$. Suppose that $p \geq 3$. Considering $S^{\prime}$ as a matrix with entries in $\bar{F}^{2 \times 2}$, let $S_{0}$ be the principal submatrix obtained from $S^{\prime}$ by deleting the first and the $v$ th rows and columns. Using the induction assumption,

$$
\operatorname{rank}\left(S^{\prime}-\lambda I_{2 p}\right) \geq \operatorname{rank}\left(S_{0}-\lambda I_{2 p-4}\right)+\operatorname{rank} S_{1, v}^{\prime} \geq p .
$$

Lemma 13 Suppose that $f_{r}(x)$ is irreducible of degree 2. If $\rho<n / 2$ and $\rho$ is odd, then there is no matrix $X \in F^{n \times n}$ such that $R_{\bar{F}}(X A-A X)=\rho$.

Proof. Suppose that $R_{\bar{F}}(X A-A X)=\rho$, where $X \in F^{n \times n}, \rho<n / 2$ and $\rho$ is odd. Take $\lambda \in \bar{F}$ such that $\operatorname{rank}\left(X A-A X-\lambda I_{n}\right)=\rho$. As $\rho<n / 2$, it can be deduced that $\lambda \in F$.

According to [6], the eigenvalues of $X A-A X$ can be joined in pairs so that the sum of the two eigenvalues of each pair is 0 . Therefore $2 \lambda=0$. Assuming that $F$ has characteristic different from 2, we have $\lambda=0$ and $\operatorname{rank}(X A-A X)=\rho$, what contradicts Lemma 4 .

Suppose that the companion matrix of $f_{r}(x)$ has the form (2).

Suppose that $b \neq 0$. As $A$ has the form $C \oplus \cdots \oplus C, X A-A X$ is a $\star-$ matrix. According Lemmas 4 and 12, the equality $\operatorname{rank}\left(X A-A X-\lambda I_{n}\right)=\rho$ is impossible.

Finally, suppose that $F$ has characteristic 2 and that $b=0$. Take

$$
Y=\left[\begin{array}{ll}
1 & 0 \\
\lambda & 1
\end{array}\right] \oplus \cdots \oplus\left[\begin{array}{ll}
1 & 0 \\
\lambda & 1
\end{array}\right] \in F^{n \times n}
$$

Then $Y A-A Y=\lambda I_{n}$ and $(X-Y) A-A(X-Y)=X A-A X-\lambda I_{n}$ has rank $\rho$, what contradicts Lemma 4 .

Bearing in mind Lemma 3, it is clear that Theorem 1 follows, immediately, from the following lemma.

Lemma 14 Let $F$ be a field such that all the irreducible polynomials in $F[x]$ have degree $\leq 2$. Let $A \in F^{n \times n}, \rho \in\{0, \ldots, n-1\}$.

The following statements are equivalent:

$\left(a_{14}\right)$ There exists a nonsingular matrix $X \in F^{n \times n}$ such that $R_{\bar{F}}(X A-$ $A X)=\rho$.

$\left(b_{14}\right)$ There exists $X \in F^{n \times n}$ such that $R_{\bar{F}}(X A-A X)=\rho$. 
$\left(c_{14}\right)$ One of the following conditions holds:

$\left(i_{14}\right) f_{r}(x)$ is irreducible of degree 2 and $\rho$ is even.

(ii $\left.i_{14}\right) f_{r}(x)$ is irreducible of degree 2 and $\rho \geq n / 2$.

(iii $\left.i_{14}\right) f_{r}(x)$ is not irreducible of degree 2 and $\rho \leq 2 R_{\bar{F}}(A)$.

Proof. Suppose that $\left(b_{14}\right)$ is satisfied. Suppose that $f_{r}(x)$ is irreducible of degree 2. According to Lemma 13, $\rho$ is even or $\rho \geq n / 2$. Now suppose that $f_{r}(x)$ is not irreducible of degree 2 . Then, using Lemma 4 ,

$$
\rho=R_{\bar{F}}(X A-A X) \leq \operatorname{rank}(X A-A X) \leq 2 R_{\bar{F}}(A) .
$$

Conversely, suppose that $\left(c_{14}\right)$ is satisfied, in order to prove $\left(a_{14}\right)$. If one of the conditions $\left(i_{14}\right),\left(i i i_{14}\right)$ is satisfied, then, according to Lemma 11, $\left(a_{14}\right)$ holds. Now suppose that $f_{r}(x)$ is irreducible of degree 2 and $\rho \geq n / 2$. Let $s:=$ $n-\rho \leq n / 2=r$. We have $A=C \oplus \cdots \oplus C$, where $C=C\left(f_{1}\right)=\cdots=C\left(f_{r}\right)$. Let $Y \in F^{2 \times 2}$ be a nonsingular matrix such that $Y C-C Y$ is nonderogatory. Let $\lambda_{1}, \lambda_{2} \in \bar{F}$ be the eigenvalues of $Y C-C Y$. Let $X_{1}=Y \oplus \cdots \oplus Y \in F^{2 s \times 2 s}$.

If $s=r$, let $X=X_{1}$.

If $s<r$, let $A_{1}=C\left(f_{1}\right) \oplus \cdots \oplus C\left(f_{s}\right), A_{2}=C\left(f_{s+1}\right) \oplus \cdots \oplus C\left(f_{r}\right)$. According to Lemma 9 , there exists a nonsingular matrix $X_{2} \in F^{2 s \times 2 s}$ such that $X_{2} A_{2}-A_{2} X_{2}$ is nonderogatory and does not have any eigenvalue in $\left\{\lambda_{1}, \lambda_{2}\right\}$. Let $X=X_{1} \oplus X_{2}$.

For any value of $s$, it is not hard to deduce that

$$
\rho=R_{\bar{F}}(X A-A X)=\operatorname{rank}\left(X A-A X-\lambda_{1} I_{n}\right)
$$

\section{References}

[1] I. Cabral, Matrices with prescribed submatrices and number of invariant polynomials, Linear Algebra Appl. 219 (1995), 207-224.

[2] R. Guralnick and C. Lanski, The rank of a commutator, Linear Multilin. Algebra 13 (1983), 167-175.

[3] T. J. Laffey, A basis theorem for matrix algebras, Linear Multilin. Algebra 8 (1980), 183-187. 
[4] M. G. Marques, The number of invariant polynomials of a matrix with prescribed off-diagonal blocks, Linear Algebra Appl., to appear.

[5] M. G. Marques, F. C. Silva and Zhang Yu Lin, The number of invariant polynomials of a matrix with prescribed complementary principal submatrices, Linear Algebra Appl., to appear.

[6] E. A. Martins and F. C. Silva, Eigenvalues of matrix commutators, Linear Multilin. Algebra 39 (1995), 375-390.

[7] G. N. Oliveira, E. M. Sá and J. A. Dias da Silva, On the eigenvalues of the matrix $A+X B X^{-1}$, Linear Multilin. Algebra 5 (1977), 119-128.

[8] E. M. Sá, The rank of the difference of similar matrices, Portugaliae Math. 46 (1989), $177-187$.

[9] F. C. Silva, On the number of invariant polynomials of the matrix $X A X^{-1}+B$, Linear Algebra Appl. 79 (1986), 1-21.

[10] F. C. Silva, On the number of invariant polynomials of partially prescribed matrices, Linear Algebra Appl. 197 (1994), 709-754.

[11] F. C. Silva and W. So, Possible numbers of invariant polynomials for the difference of two similarity classes, Linear Multilin. Algebra, to appear. 